\title{
Der PlanBuden-Prozess: das Interesse des Stadtteils als Grundlage von Planung
}

Renée Tribble, Patricia Wedler

\section{Zwischen hipper Wohnlage und touristischem Ballermann}

Dem PlanBuden-Prozess geht ein ziviler Protest eines emanzipierten Stadtteils voraus, der 1989 noch ,der mit Abstand finanziell ärmste Stadtteil“ (Manos 1989: 11, in Wischmann 2016: 30) in Hamburg war: St. Pauli. Jedoch ist seit Ende der 1990er-Jahre vermehrt ein Zuzug an Akademiker*innen und Haushalten mit höheren Einkommen zu verzeichnen und damit verbunden auch eine erhebliche Mietpreissteigerung. Im Vergleich zum Jahr 2000 lagen die Angebotsmieten infolge von Investitionen und Modernisierungen 2011 um 51 \% höher (Wischmann 2016: 24). Auch die Preise für Eigentumswohnungen weisen eine rasante Steigerung auf. 2004 kostete der Quadratmeter bei Eigentumswohnungen 1.912€ (Hamburg: $2.026 €$, Statistikamt Nord 2004: 18), 2016 bereits $4.652 €$ und damit knapp $700 €$ mehr als der durchschnittliche Eigentumspreis für Wohnungen in Hamburg (3.965€, vgl. Statistikamt Nord, Stadtteilprofile 2018: 29). Damit orientieren sich die Preise an den Preisen in guter bis sehr guter Wohnlage, etwa in den Gründerzeitvierteln rund um die Alster, die bei ca. 5.000€ pro Quadratmeter liegen. Dennoch ist St. Pauli immer noch ein Rotlichtbezirk, stark beworbene Ausgehmeile und Touristenattraktion, vom Hamburger Stadtmarketing als „der wahrscheinlich bekannteste Stadtteil Hamburgs“" (Otremba 2018) betitelt.

Der Widerspruch zwischen begehrter, hipper Wohnlage und touristischer Ballermann-Realität hat seine Folgen. Ein- und Auszüge sind häufig; viele neu Zugezogene verlassen den Kiez binnen zwei Jahren. Die hohe Fluktuation trägt wiederum ihren Teil zu steigenden Mieten bei: „91\% der zwei Jahre alten Mietverhältnisse liegen über dem Mittelwert des Mietspiegels 2009“ (Bezirksamt Hamburg-Mitte 2011: 7). Alteingesessene Mieter*innen finden bei Verlust der Wohnung nur schwer eine neue im Quartier. Eine große Sorge der Nachbarschaft ist, dass der Zusammenhalt untereinander verloren gehen könnte. Bislang ist der Austausch in der Nachbarschaft hoch (siehe Abb. 1). 
Wie ist der Kontakt unter den Nachbarlnnen?

$(\mathbb{N}=727)$
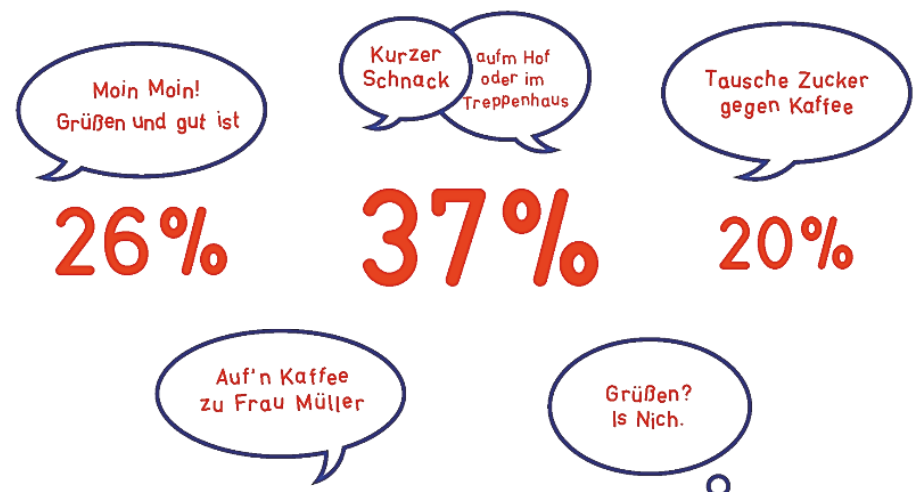

$15 \%$

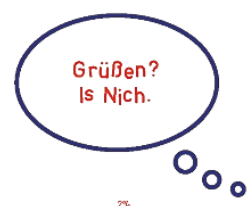

Abbildung 1: $\quad$ Auswertung PlanBude: Auf eine Tasse Kaffee - wie ist es in Ihrer Nachbarschaft?

Quelle: tumulti.

Die Politik reagierte auf diese Entwicklung mit dem Versuch eines Milieuschutzes. Nachdem die drei Sanierungsgebiete im nördlichen St. Pauli (St. Pauli-Nord S 1/Schilleroper (1980-2014), St. Pauli Nord S 3/Karolinenviertel (1988-2012) und St. Pauli S 5/Wohlwillstraße (1997-2014)) alle ausgelaufen waren, wurde für sämtliche Wohngebiete des Stadtteils im Februar 2012 eine soziale Erhaltungsverordnung aufgestellt. Durch die soziale Erhaltungsverordnung soll die Umwandlung zu Eigentumswohnungen erschwert werden und die Mietsteigerung moderat bleiben - in der Realität erweist sich diese Maßnahme jedoch als wenig wirksam, es finden sich Mittel und Wege dies zu umgehen, denn die Nachfrage auf dem Wohnungsmarkt insbesondere in den zentralen Lagen ist in Hamburg nach wie vor hoch.

\section{Eine Nachbarschaft organisiert sich}

Als 2009 die Esso-Häuser, ein Gebäudekomplex aus den 1960er-Jahren, prominent an der Reeperbahn gelegen und bestehend aus Gewerbeeinheiten, Mietwohnungen und einer namensgebenden Tankstelle, den Eigentümer wechseln und kurz danach den ca. 100 Mietparteien Schreiben für neue Mietverträge zugehen, sind 
einige Bewohner*innen aufmerksam. Nach Rücksprache mit dem „Mieter-helfenMietern"-Anwaltsverein ist schnell klar: Wer die neuen Verträge unterschreibt, verliert seine Ansprüche, v. a. als langjährige Mieterin bzw. langjähriger Mieter. Im Fall einer Kündigung keine gute Ausgangslage - zumal die neue Eigentümerin, die Bayerische Hausbau, von Abriss und Neubauplänen spricht.

Mithilfe der Gemeinwesenarbeit (GWA) St. Pauli werden sämtliche Mieter*innen informiert; die Initiative Esso-Häuser aus Bewohner*innen, Nachbar*innen und Gewerbetreibenden gründet sich im Jahr 2010 (vgl. Initiative Esso Häuser 2010) . Ihr langjähriger Kampf gegen den Abriss und für eine Sanierung der Häuser endet abrupt mit der plötzlichen Evakuierung der Gebäude im Dezember 2013. Ein Wackeln der Wände sei spürbar gewesen, melden zwei Bewohner telefonisch der Polizei; ein paar Stunden später ist das gesamte Ensemble inklusive Clubs, Bars und Hotel evakuiert. Die Bewohner*innen werden mit Bussen zu einer Notunterkunft gebracht. Kurz danach wird eine Recht-auf-Stadt-Demonstration von der Polizei gestoppt und es kommt auf der Reeperbahn unmittelbar vor den Esso-Häusern, die zum Symbol der Gentrifizierungsprozesse auf St. Pauli werden, zu Ausschreitungen. Trotz fast täglicher Demonstrationen, auch vor den EssoHäusern, wird dem Abriss-Antrag, der bereits im August 2013 von der Bayerischen Hausbau eingereicht worden war (vgl. Hagn 2013), am 21. Januar 2014 (Bezirksamt Hamburg-Mitte 2014a) stattgegeben.

Aufgrund der prekären Situation laden Initiativen aus dem Stadtteil zu einer Stadtteilversammlung im Ballsaal des Millerntorstadions ein. Aus der Versammlung mit mehr als 400 Teilnehmer*innen geht die Ballsaalresolution hervor, die zu den Esso-Häusern fordert:

„Es gibt einen von unten organisierten, demokratischen Planungsprozeß. St. Pauli hat längst gezeigt, dass das lokale Know-How interessante, soziale städtebauliche Lösungen entwickeln kann, die der hervorgehobenen Bedeutung des Geländes am Spielbudenplatz gerecht werden. Der Runde Tisch zu Park Fiction 1997/98 unter Senator Mirow belegt, dass das möglich ist - wenn der politische Wille da ist. [...] Wir laden alle Anwohner_innen und Interessierten dazu ein, ihr Wissen und ihre Ideen in diesen Prozess einzubringen - und werden umgehend damit beginnen, die Planung selbst in die Hand zu nehmen“(St. Pauli Selber Machen 2014).

Die aus der Stadtteilversammlung entstehende Planungs-AG wird Ausgangspunkt der „PlanBude“. Die PlanBude ist zunächst ein Konzept der Planungs-AG, welches dem Bezirksamtsleiter im Rahmen eines von ihm ausgegangenen Gesprächsangebots vorgestellt wird. Das Konzept sieht vor, dass mehrere Container am Standort für ein Jahr einen Ort schaffen sollen, an dem der Stadtteil selbst den Planungsprozess für einen Neubau der Esso-Häuser durchführt. Sherry Arnsteins „eight rungs of a ladder of participation“ stehen symbolisch für den anvisierten 
Prozess (vgl. Arnstein 1969). In ihrer Pressekonferenz, in der sie im April 2014 die Mitsprache des Stadtteils an der Planung fordert (siehe Abb. 2), fordert die Planungs-AG zivilgesellschaftliche Macht („Degrees of Citizen Power") statt Alibi-Beteiligung („,Degrees of Tokenism“). In weiteren Gesprächen mit Vertreter*innen der Planungs-AG und des Bezirks konkretisiert sich das Konzept. Im Sommer 2014 wird schließlich der offizielle Beteiligungsprozess als frühzeitige Beteiligung an die eigens dafür gegründete PlanBude GbR vergeben.

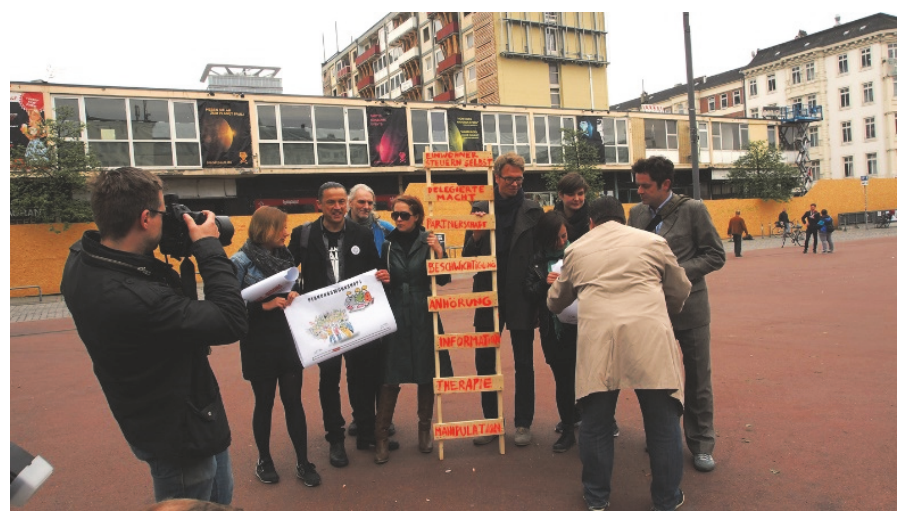

Abbildung 2: $\quad$ Entscheidungsmacht statt Alibibeteiligung! Pressekonferenz der Planungs-AG mit der „Ladder of Participation“ im April 2014

Foto: M. Czenki, 2014

\section{Die Carte blanche ausspielen}

Gegenüber formeller Beteiligung in Bebauungsplanaufstellungsverfahren ist der Zeitpunkt der Beteiligung im PlanBuden-Prozess in der Tat frühzeitig. Der Beteiligungsprozess geht den weiteren Planungsschritten, einem städtebaulichen Wettbewerb, gefolgt von einem hochbaulichen Gutachterverfahren und der Aufstellung eines neuen Bebauungsplans, voraus. „Der Stadtteil [soll] in einem breit angelegten Mitwirkungsprozess einbezogen werden und wichtige Grundlagen für den anschließenden Wettbewerb liefern“" (Bezirksamt Hamburg-Mitte 2014b). Der damalige Bezirksamtsleiter Andy Grote stellt auf der Pressekonferenz im Juli 2014 die Anforderungen an den angestrebten Prozess heraus:

,[...] Auf dem Grundstück soll ein echtes, unverwechselbares Stück St. Pauli entstehen. Damit das gelingt, wollen wir gerade an diesem umkämpften und symbolträchtigen Vorhaben eine neue Qualität von Beteiligungskultur erproben. Eine vergleichbar 
frühzeitige, umfassende und eng am Stadtteil ausgerichtete Beteiligung hat es bisher in Hamburg nicht gegeben " (ebd.).

Die entscheidende Formulierung ist jedoch in der Präambel des zwischen der PlanBude und dem Bezirksamt Hamburg-Mitte geschlossenen Vertrags festgehalten:

„Um dieses zu erreichen, beabsichtigt das Bezirksamt einen Planungsprozess durchzuführen, der vom Stadtteil intensiv begleitet und mitgestaltet wird. Die Bedarfe und Interessen des Stadtteils sollen in den Planungsprozess einfließen und im Rahmen der Abwägung der verschiedenen Belange innerhalb der planerischen Gestaltungsspielräume ein eigenes Gewicht erhalten“" (FHH 2014).

Die explizite Formulierung, dass die Interessen des Stadtteils ein eigenes Gewicht erhalten sollen, ist zum einen die Voraussetzung, um die unterschiedlichen Interessen der drei Akteurssphären der Stadtentwicklung - Wirtschaft, Staat und Zivilgesellschaft (Selle 2013) überhaupt verhandeln zu können, und zum anderen Garant für die Glaubwürdigkeit des Prozesses. Die Ergebnisse des Beteiligungsprozesses können und sollen Grundlage der weiteren Planung werden, ebenso wie die wirtschaftlichen Interessen der Eigentümerin und Investorin Bayerische Hausbau eine Rolle spielen werden. Häufig wird jedoch das Interesse nur einseitig ermittelt, wenn es z. B. in Form eines konkreten Bebauungsvorschlags vonseiten des Investors vorliegt. Im Fall des PlanBuden-Prozesses wird das Stadtteilinteresse jedoch zu einem Zeitpunkt ermittelt, in dem aus politischer Sicht noch eine Carte blanche besteht. Denn es gibt zwar städtebauliche Studien, die die mögliche bauliche Ausnutzung, wie viel auf dem Grundstück gebaut werden könnte, nachweisen; für die Umsetzung ist aber ein neuer Bebauungsplan erforderlich - und dieser benötigt eine positive politische Entscheidung. Im PlanBuden-Prozess erfolgt die Entscheidung über die Aufstellung eines neuen Bebauungsplans erst sechs Monate nach dem hochbaulichen Gutachterverfahren im Februar 2016 (Bezirksamt Hamburg-Mitte 2016) und damit erst, nachdem das Interesse des Stadtteils ermittelt, übersetzt, verhandelt und als Grundlage in die Aufgabenstellung des Wettbewerbs geflossen ist.

\section{Das Interesse des Stadtteils formulieren}

Im Vergleich mit anderen informellen Verfahren sticht die Dauer und Intensität des Beteiligungsprozesses heraus. Die PlanBude hatte viereinhalb Monate jeweils sechs Tage die Woche von 16 bis 21 Uhr geöffnet. Allein dieser Zeitraum stellt eine Besonderheit dar. Die Präsenz und unmittelbare Zugänglichkeit vor Ort in Form der Container mit Dachterrasse trug zum einem zum Vertrauen des Stadtteils in den Prozess bei und zum anderen wurde diese Möglichkeit des Zugangs zu einer 
Gelegenheit des Alltags. Die Selbstverständlichkeit, mit der die zwei PlanBudenContainer im Stadtraum platziert sind, - auf der einen Seite zum Touristenstrom der Reeperbahn, auf der anderen zum Alltagsweg der Bevölkerung und unmittelbar am Ort der ehemaligen Esso-Häuser - versinnbildlicht die Forderung der Ballsaalresolution sprichwörtlich. Das am Fenster platzierte Wunscharchiv zeigt zudem den Fortschritt des Prozesses an - im Vorbeigehen lässt sich mit einem Blick auf das Archiv der zunehmende Füllstand wie ein Barometer ablesen. Die Inbesitznahme des Ortes bei gleichzeitiger andauernder Sichtbarkeit führt dazu, dass die Container mittlerweile symbolisch für die Absicherung des Stadtteilinteresses im weiteren Planungsprozess stehen.

Die vom PlanBuden-Team (siehe Abb. 3) entwickelten Tools, die Workshops zu verschiedenen Themensträngen wie „Günstig bauen - günstig wohnen“, zur sozialen Frage, wer eigentlich verdrängt wird, zu aktuellen Formen des Wohnens wie „Mehr haben durch teilen“ oder auch zur Musik- und Sexkultur sowie aktivierende Haustürgespräche und der an alle Haushalte St. Paulis versandte Fragebogen diversifizieren die Möglichkeiten, sich einzubringen, und erreichen darüber hinaus eine breite und hohe Zahl an Anwohner*innen. Ein Ziel des PlanBuden-Prozesses war es, „diejenigen [zu] erreichen, die von der Gestaltung der Stadt am meisten ausgeschlossen sind“" (PlanBude o. J.). Die unterschiedlichen Formate der PlanBude versuchen, durch Vielfalt, Detaillierung und gleichzeitige Offenheit eine möglichst große Bandbreite an Themen für die Neuentwicklung des Areals abzudecken - und gleichzeitig der eigenen Kreativität nicht nur Freiraum zu lassen, sondern explizit dazu einzuladen und anzuregen, diese einzusetzen - egal ob geschrieben, gemalt, gezeichnet, gebaut oder in Gesprächen am großen Tisch formuliert. Einige Prämissen der Arbeitsweise fasst PlanBude in ihrem in dérive 2015 veröffentlichten Artikel zusammen: „Sei neugierig [...] Sei vor Ort (verräumliche Dein Begehren) $[\ldots]$ Sei offen [...] Rede mit allen [...] Sei ernst. Sei verspielt. Erfinde neue Methoden. [...] Sprich mit vielen unterschiedlichen Stimmen“ (Schäfer et al. 2015: 37 ff.).

Im Kern ist der Beteiligungsprozess der PlanBude eine Wunschproduktion, die „utopischen Überschuss“ produziert, sich von Deleuze und Guattari ableitet und erstmals im Kunstprojekt im öffentlichen Raum Park Fiction stattgefunden hat (vgl. PlanBude \& Petruschat 2016: 368). Dieses erfolgreich realisierte Projekt hat auch zu dem Mut beigetragen, sich auf einen solchen Prozess wie den der PlanBude mit offenem Ausgang einzulassen - sowohl vonseiten des Stadtteils als auch vonseiten des Bezirks. Durch die Wunschproduktion wird das eingeschriebene Erfahrungswissen vor Ort für alle sichtbar und für jede*n nutzbar - denn der von Anwohner*innen geplante Park Fiction ist gebaut. 
„Die PlanBude ist ein trans-disziplinäres Team aus den Feldern Kunst, Architektur, Urbanistik, sozialer Stadtteilarbeit, Musik und Kulturwissenschaft. Die PlanBude wurde 2014 aus einer unabhängigen Stadtteilversammlung ,,St. Pauli selber machen “im Ballsaal des FC St. Pauli heraus gegründet, um die Wunschproduktion für die neuen EssoHäuser zu organisieren - und damit einen neuen Ansatz zu entwickeln, wie Stadt anders geplant und gebaut werden kann und muss. Der Großteil des [...] Teams lebt auf St. Pauli. [...] im Auftrag des bezirklichen Baudezernats [...] öffnete die PlanBude ihre Türen direkt am Bauplatz, im Herzen St. Paulis, direkt an Reeperbahn und Spielbudenplatz.

[...] das Team [hat] eine breite Palette an Zugängen zum Planungsprozess entwickelt: Von Haustürgesprächen und flächendeckend verteilten Fragebögen in fünf Sprachen, zum vor Ort installierten Planungscontainer, bis zu anregenden, künstlerischen und anspruchsvollen Tools wie das Knetmodell (M 1:500), das Legomodell (M 1:150), Nachtkarten, Lesungen und Workshops in den umliegenden Kneipen, Lokalen, sozialen Einrichtungen und Kaschemmen.

Die ungewöhnliche Herangehensweise der PlanBude zeigt ein neues Rollenmodell an: Kunst, Architektur, Planung und Soziale Arbeit, die sich in enger Zusammenarbeit inhaltlich, nachbarschaftlich und politisch positionieren und so die Grundlage und Methodik entwickeln, damit das Wissen der Vielen die Stadt gestalten kann. Dabei unterscheiden sich die von der PlanBude ermittelten Ergebnisse stark von dem, was in der Politik befürchtet wurde: Hohe Dichte wurde akzeptiert, wenn dafür langfristig abgesicherter Wohnraum im Zentrum entsteht; kein monolithischer Block, sondern lieber ein paar hohe Häuser und dafür mehr Varianz; wenn die Dichte so hoch ist, sollte es zum Ausgleich benutzbare Dächer, Dachlandschaften geben, die unterschiedlich nutzbar sind; ein zugänglicher, für alle benutzbarer urbaner Sockel voller Läden, Einrichtungen und mit subkulturellem oder sozialem ,,Mehrwert" für das Viertel; Orte zum ,, St. Pauli selber machen “.

\section{Abbildung 3: $\quad$ PlanBude (o. J.)}

Im Fall des PlanBuden-Prozesses wird der aus den über 2.300 entstandenen Beiträgen abgeleitete St. Pauli Code zum Ausdruck des Erfahrungswissens. Das geballte Wissen - in Zeichnungen, Zitaten, Fragebögen, Modellen etc. ausgedrückt und festgehalten - wird zunächst vom Team der PlanBude ausgewertet, geclustert, in mehreren Hundert Folien zusammengestellt und im Stadtteil präsentiert. In einem zweiten Schritt werden die Aussagen kondensiert und zu einer Position des Stadtteils zugespitzt. Diese bildet die Grundlage für die Verhandlungsworkshops mit Eigentümerin und Bezirk, in denen nun die Interessen aller drei an Stadtentwicklung beteiligten Akteurssphären - Wirtschaft, Staat und Zivilgesellschaft (Selle 2013) - einander gegenüberstehen. In dem daraus hervorgegangenen Eckpunktepapier sind die mit Eigentümerin und Bezirk vereinbarten Leitlinien für die Neubebauung festgehalten. 


\section{Geteilte Verantwortung: von der Übersetzung zur Verankerung in der Stadt}

Nach der Übersetzung in eine mit der Eigentümerin und dem Bezirk abgestimmte Auslobung zeugt der einstimmige Beschluss der Jury im September 2015 für den städtebaulichen Gewinnerentwurf von der Überzeugungskraft, die durch diesen Prozess entstanden ist. Dem Entwurf von NL Architecture und BeL Sozietät für Architektur gelingt es, die unterschiedlichen und teils widersprüchlichen Interessen städtebaulich zu integrieren. Die im St. Pauli Code enthaltenen Kriterien wie z. B. Kleinteiligkeit, Widersprüchlichkeit, urbaner Sockel und Freiräume ohne Konsumzwang spiegeln sich in der räumlichen Übersetzung des städtebaulichen Entwurfs mit zwölf unterschiedlichen Gebäuden von drei bis 13 Geschossen, öffentlich zugänglicher Dachlandschaft und einer neuen Passage, die das Erdgeschoss um weitere Schnittstellen zum öffentlichen Raum bereichert, wider (siehe Abb. 4).

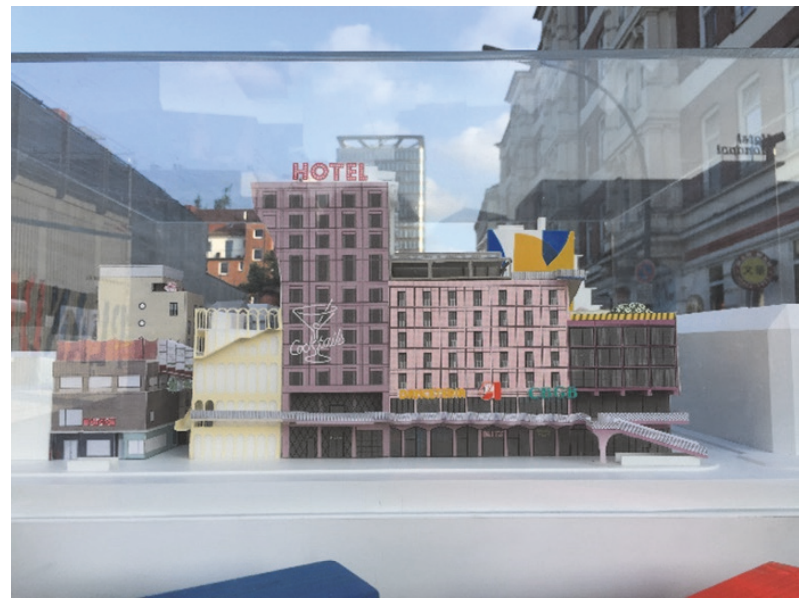

Abbildung 4: $\quad$ Der überarbeitete und weiterentwickelte hochbauliche Entwurf für den Neubau der Esso-Häuser

Quelle: PlanBude, Renée Tribble.

Ein Teil der Prozessergebnisse ist damit baulich-räumlich übersetzt; ein weiterer großer und bedeutender Teil muss politisch festgesetzt und gesichert werden - im Rahmen eines städtebaulichen Vertrags, der dem neuen Bebauungsplan vorausgehen wird. Auch in der Realisierung wird es den ausgesprochenen Willen aller Beteiligten erfordern, um die Herausforderungen zu meistern, die mit der ausgespro- 
chen hohen Dichte, der nutzungsgemischten Bebauung, den wirtschaftlichen Interessen und den Normen der Gesetze einhergehen.

Bislang finden sich alle Akteur*innen mit ihren Interessen im Projekt wieder - und zugleich wurde jeweils gegenüber der eigenen Sphäre die Verantwortung übernommen, dass sich diese auch in den realisierten Gebäuden und in der tatsächlichen Nutzung wiederfinden werden. Bislang, so scheint es, ist dies der Kitt, der den Prozess zusammenhält.

Im Rückblick erscheinen für die Verankerung des Stadtteilinteresses im Planungsprozess folgende Punkte wesentlich:

- frühzeitiger Beteiligungsprozess, solange noch Entscheidungsmacht besteht,

- Zusicherung der Relevanz der Ergebnisse des Beteiligungsprozesses in der weiteren Planung,

- Dauer und Tiefe des Beteiligungsprozesses,

- gleichberechtigte Stellung des Stadtteilinteresses neben den Interessen von Planung und Politik sowie Eigentümer*innen und Investor*innen,

- Ableitung von grundsätzlichen Prinzipien und damit prüfbarer Kriterien für die zukünftige Entwicklung.

Dafür braucht es hohes Engagement und sowohl Übernahme als auch Abgabe von Verantwortung durch die bzw. von den beteiligten Akteur*innen. So war die PlanBude in der Phase des Beteiligungsprozesses in der Verantwortung; der Bezirk dagegen nahm eine kommentierende Rolle und die Eigentümerin und Investorin eine beobachtende Rolle ein. D. h., sie war ohne Einfluss auf den von der PlanBude konzipierten und durchgeführten Beteiligungsprozess. Die Zivilgesellschaft hat in dieser Phase einen Rahmen erhalten, um ihre Interessen zu artikulieren und diese - zugespitzt und übersetzt durch die PlanBude - in den Planungsprozess einzubringen. Erst anschließend, nachdem sämtliche Beiträge ausgewertet, übersetzt und im Stadtteil vorgestellt und diskutiert worden waren, erfolgte die Auseinandersetzung und Verhandlung mit dem Bezirk und der Eigentümerin. Dieser Moment ist wesentlich für ernst gemeinte Teilhabe, damit Beteiligung nicht zur Alibihandlung wird (vgl. Abb. 2 oben). Dafür müssen Prozesse, Zeiträume und Akteur*innen gefunden werden, die diese notwendige und intensive Auseinandersetzung mit einer städtebaulichen Entwicklung, die ein Quartier wesentlich prägt, überhaupt ermöglichen.

Als „Good-Practice“-Beispiel wirft der PlanBuden-Prozess die Frage auf: Was ist davon auf andere Projekte übertragbar?

Personelle Hintergründe und Erfahrungswissen sind stets individuell, persönliche Betroffenheit und die Identifikation mit dem Stadtteil zählen jedoch zu den typischen quartiersbezogenen Faktoren, die auch an anderen Orten mit hoher lokaler Identifikation und bestehenden und gut funktionierenden Netzwerken als an 
den Stadtteil geknüpft, nicht aber als einzigartig für St. Pauli bezeichnet werden können. Die Motivation des Engagements ist jedoch nicht nur mit dem Stadtteil, sondern auch eng mit der Historie des Projekts und dessen symbolhaftem Charakter verbunden. Die Hoheit über einen neu aufzustellenden oder zu verändernden Bebauungsplan und damit die Entscheidungsmacht obliegt unabhängig von der Vorgeschichte immer gewählten und demokratisch legitimierten Politiker*innen. Die Prinzipien der Frühzeitigkeit, der Intensität des Beteiligungsprozesses und der Relevanz der Ergebnisse können ebenfalls in anderen Situationen zur Geltung gebracht werden, vorausgesetzt die beteiligten Akteur*innen haben den Mut dazu. Denn dazu gehört auch ein großes Vertrauen, dass mit dem Wissen der Vielen und dem Alltagserfahrungswissen nicht nur eine lokale Spezifik verbunden ist, sondern auch etwas, was nicht nur den partikularen Interessen einzelner Akteur*inne, sondern dem ganzen Stadtteil entspricht. David Harvey beschreibt dies mit dem „positive moment“ (Harvey 1996: 236):

„In other words, in this view foundational values and beliefs were discovered in particular struggles and then translated onto a broader terrain of conflict. It seems to me that the notion of community, viewed in this way, can be a positive moment within a political process. However, it is only a positive moment if it ceases to be an end in itself, ceases to be a thing which is going to solve all of our problems, and starts to be a moment in this process of broader construction of a more universal set of values which are going to be about how the city is going to be as a whole."

Ein kritischer Punkt wird also sein, ob es gelingt, zu universellen Werten für die zukünftige Stadtentwicklung zu kommen, auf die man sich in einem politischen Prozess verständigen kann. Aber auch wenn es gelingt, über Partikularinteressen - zu denen auch wirtschaftliche Interessen von Projektentwickler*innen zählen hinauszugehen und eine auf der Basis eines breiten Verständnisses durch eine tiefgehende Beteiligung erarbeitete Vorstellung von Stadt in Stadtentwicklung einzuschreiben, so bleiben weitere wesentliche Aspekte offen, für die praxistaugliche Lösungsansätze wünschenswert sind: Wie können die asymmetrischen Machtverhältnisse ausgeglichen werden, die z. B. durch die unterschiedliche ökonomische Macht der Akteur*innen entstehen? Müssen die wirtschaftlichen Interessen nur dann zurückstehen, wenn es zivilgesellschaftliches Engagement vor Ort gibt? Was ist, wenn dieses noch nicht vorhanden ist? Und wie kann man in anderen Prozessen von außen aktivieren, ohne jemanden zu bevormunden? Ein wesentlicher Aspekt wird allzu oft bei der Frage nach Übertragbarkeit vergessen: „, [P]ersonal engagement is not a service“ (PlanBude \& Brahm 2018: 117) - dies zu verstehen, wird grundlegend für zivilgesellschaftliche Prozesse in der Stadtentwicklung sein. Wenn auch an dieser Stelle nicht alle Fragen beantwortet werden können, so können Beteiligungsprozesse, die auf „Citizen Power“ zielen, doch auch wieder zu 
mehr Vertrauen in das kollektive Wissen und dessen Verankerung in Stadtentwicklungsprozessen beitragen.

\section{Literatur}

Arnstein, S. R. (1969): A Ladder of Citizen Participation. In: JAIP (35) 4: 216-224.

Bezirksamt Hamburg-Mitte, Dezernat Wirtschaft, Bauen und Umwelt, Fachamt Stadt- und Landschaftsplanung (2011): BEGRÜNDUNG zum Erlass einer Sozialen Erhaltungsverordnung gemäß § 172 Abs. 1 Satz 1 Nr. 2 Baugesetzbuch für St. Pauli. Abrufbar unter: http://www.hamburg.de/contentblob/6115380/83950d5d08d5d8bfadd94ed5ec 8ba594/data/sozerhalt-stpauli-beg-dl.pdf. Letzter Zugriff: 21.4.2018.

Bezirksamt Hamburg-Mitte, Dezernat Wirtschaft, Bauen und Umwelt, Fachamt Bauprüfung (2014a): Abbruch aller Gebäude auf dem Grundstück Kastanienallee 14, Spielbudenplatz 5, 5a, 5b, 5c, 7, 9, 11, 13, Taubenstraße 22, einschließlich der gesamten Tiefgarage. Transparenzportal Hamburg, Genehmigung nach HBauO. Abrufbar unter: http://transparenz.hamburg.de/. Datenlizenz Deutschland - Namensnennung Version 2.0. Letzter Zugriff: 20.4.2018.

Bezirksamt Hamburg-Mitte (2014b): Bürgerbeteiligung Grundstück Esso-Häuser startet. Pressemitteilung vom 22.07.2014. Abrufbar unter: http://www.hamburg.de/pressearchiv-fhh/4346988/buergerbeteiligung-esso-haeuser/. Letzter Zugriff: 20.4.2018.

Bezirksamt Hamburg-Mitte, Bezirksversammlung, Stadtplanungsausschuss (2016): Bebauungsplan-Entwurf St. Pauli 45 (Spielbudenplatz 5-13) Zustimmung zur Einleitung des Bebauungsplanverfahrens. Drucksache 21-1909 vom 15. Februar 2016. Abrufbar unter: https://sitzungsdienst-hamburg-mitte.hamburg.de/bi/vo021.asp?VOLFDNR=100 7257. Letzter Zugriff: 20.4 .2018

Freie und Hansestadt Hamburg (FHH) vertreten durch das Bezirksamt Mitte. 7.11.2014, Vertrag mit PlanBude GbR, Präambel.

Hagn, S. (2013): Die Bayerische Hausbau bekräftigt ihr Angebot für die Mieterinnen und Mieter am Spielbudenplatz. Pressemitteilung Bayerische Hausbau vom 14. August 2013. Abrufbar unter: http://www.wohnen-am-spielbudenplatz.de/fileadmin/user_upload/presse/130814_Die_Bayerische_Hausbau_bekraeftigt_ihr_Angebot_fuer_die_M ieterinnen und Mieter_am Spielbudenplatz.pdf. Letzter Zugriff: 20.4.2018

Harvey, D. (1996). Contested Cities: Social Process and Spatial Form. In F. LeGates, Richard T.; Stout (Ed.), The City Reader (5th). Routledge: 230-237.

Initiative Esso Häuser (2010): Wichtige Mitteilung an alle BewohnerInnen von St. Pauli. Abrufbar unter: http://www.initiative-esso-haeuser.de/. Letzter Zugriff: 20.4.2018.

Otremba, M. (2018): Das ist Hamburg. Hamburg Marketing GmbH. Abrufbar unter: https://marketing.hamburg.de/das-ist-hamburg.html. Letzter Zugriff: 21.4.2018

Manos, H. (1989): Soziale Lagen und soziale Fragen im Stadtteil Sankt Pauli. Hamburg: Ergebnisse.

PlanBude Hamburg: Czenki, M. Katthagen, V. Röthig, C., Schäfer, C., Tribble, R. \& Zander, L. (o. J.): PlanBude - Intro - D. Abrufbar unter: http://planbude.de/planbude-intro/. Letzter Zugriff: 30.3.2018. 
PlanBude Hamburg: Czenki, M. Katthagen, V. Röthig, C., Schäfer, C., Tribble, R. \& Zander, L. (o. J.): PlanBude Konzept. Abrufbar unter: http://planbude.de/planbude-konzept/. Letzter Zugriff: 3.4.2018.

PlanBude, Petruschat, J. (2016): Wunschproduktion in und gegen die Realität: In: Fezer, J. \& Studio Experimentelles Design (Hrsg.): Öffentliche Gestaltungsberatung - Public Design Report 2011-2016. Berlin.

PlanBude Hamburg (2018): PlanBude im Gespräch mit Daniela Brahm, Exrotaprint. In: Koch, M., Rost, A., Siegmund, Y., Tribble, R. \& Werner, Y. (Hrsg.): New Urban Professions A Journey through Practice and Theory. Perspectives in Metropolitan Research 5. Berlin.

Schäfer, C.; Tribble, R.; Wedler, P.; Zander, L.; Czenki, M.; Rothig, C. (2015): „Wir nennen es PlanBude“, dérive - Zeitschrift für Stadtforschung (61): 37-40.

Selle, K. (2013): Über Bürgerbeteiligung hinaus. Stadtentwicklung als Gemeinschaftsaufgabe? Analysen und Konzepte. Detmold.

St. Pauli Selber Machen (2014): Ballsaalresolution vom 08. Februar 2014. Abrufbar unter: http://www.st-pauli-selber-machen.de/?page_id=434. Letzter Zugriff: 3.4.2018.

Statistikamt Nord (2004): Hamburger Stadtteil-Profile 2004 und Umland-Profile. Statistisches Amt für Hamburg und Schleswig Holstein. Hamburg/ Kiel. Abrufbar unter: https://www.statistik-nord.de/fileadmin/Dokumente/NORD.regional/Stadtteil_Profi le2004.pdf. Letzter Zugriff: 21.4.2018.

Statistikamt Nord (2018): Hamburger Stadtteil-Profile Berichtsjahr 2016. Statistisches Amt für Hamburg und Schleswig Holstein. Hamburg. Abrufbar unter: https://www.statistiknord.de/fileadmin/Dokumente/NORD.regional/NR19_Statistik-Profile_HH_2017. pdf. Letzter Zugriff: 21.4.2018.

Wischmann, K. (2016): Städtische Visualität und Materialität : Untersuchung stadtteilpolitischer Diskurse am Beispiel von Hamburg-St. Pauli. Wiesbaden.

Open Access Dieses Kapitel wird unter der Creative Commons Namensnennung 4.0 International Lizenz (http://creativecommons.org/licenses/by/4.0/deed.de) veröffentlicht, welche die Nutzung, Vervielfältigung, Bearbeitung, Verbreitung und Wiedergabe in jeglichem Medium und Format erlaubt, sofern Sie den/die ursprünglichen Autor(en) und die Quelle ordnungsgemäß nennen, einen Link zur Creative Commons Lizenz beifügen und angeben, ob Änderungen vorgenommen wurden.

Die in diesem Kapitel enthaltenen Bilder und sonstiges Drittmaterial unterliegen ebenfalls der genannten Creative Commons Lizenz, sofern sich aus der Abbildungslegende nichts anderes ergibt. Sofern das betreffende Material nicht unter der genannten Creative Commons Lizenz steht und die betreffende Handlung nicht nach gesetzlichen Vorschriften erlaubt ist, ist für die oben aufgeführten Weiterverwendungen des Materials die Einwilligung des jeweiligen Rechteinhabers einzuholen.

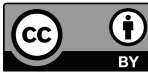

